\title{
Efficacy and safety of anti-PD-1-based therapy in combination with PARP inhibitors for patients with advanced solid tumors in a real-world setting
}

\author{
Zhaozhen $\mathrm{Wu}^{1,2,3}$ (1) Haitao Tao ${ }^{1} \cdot$ Sujie Zhang ${ }^{1} \cdot$ Xiao Wang ${ }^{4} \cdot$ Junxun $\mathrm{Ma}^{1} \cdot$ Ruixin $\mathrm{Li}^{1} \cdot$ Zhefeng Liu $^{1}$. \\ Jinliang Wang ${ }^{1}$. Pengfei Cui ${ }^{1}$. Shixue Chen ${ }^{1} \cdot$ Huang $\mathrm{Di}^{1,3} \cdot$ Ziwei Huang ${ }^{1,3} \cdot$ Xuan Zheng ${ }^{1} \cdot \mathrm{Yi} \mathrm{Hu}^{1,3}$
}

Received: 30 March 2020 / Accepted: 5 January 2021 / Published online: 19 March 2021

(c) The Author(s) 2021

\begin{abstract}
Background Rationale exists for combining immune checkpoint inhibitors and PARP inhibitors (PARPi), and results of clinical trials in ovarian cancer are promising, but data in other cancers are limited.

Method Efficacy and safety of PARPi/anti-PD-1 in advanced solid tumors were retrospectively analyzed. The efficacy measures included objective response rate (ORR), disease control rate (DCR), progression-free survival (PFS) and overall survival (OS).

Results This retrospective study included data from 40 patients. The ORR was $27.5 \%$ (95\% CI, 13.0-42.0\%), with a DCR of $85.0 \%$ (95\% CI, 73.4-96.6\%). Except four patients in first-line treatment (three with PR and one with SD), the ORR of $\geq$ second-line treatment, non-small cell lung cancer (NSCLC) and small cell lung cancer (SCLC) was $22.2 \%, 23.1 \%$ and $28.6 \%$, and the DCR was $83.3 \%, 84.6 \%$ and $71.4 \%$, separately. The median PFS of all patients, $\geq$ second-line treatment, NSCLC and SCLC was $4.6 \mathrm{~m}, 4.2 \mathrm{~m}, 4.5 \mathrm{~m}$ and $3.7 \mathrm{~m}$. The median OS was $9.4 \mathrm{~m}, 11.4 \mathrm{~m}, 12.7 \mathrm{~m}$ and $5.4 \mathrm{~m}$, respectively. Multivariable analysis revealed that BRCA1/2 mutation was positively correlated with ORR $(P=0.008)$, and LDH $\geq 250 \mathrm{U} / \mathrm{L}$ was negatively correlated with lowered DCR $(P=0.018)$, while lymphocyte number, ECOG and LDH significantly influenced both PFS and OS. We found that the possible resistant mechanisms were sarcomatous degeneration and secondary mutation, including BRCA2 truncation mutation, A2M, JAK1,T790M, KEAP1 and mTOR mutation. 37.5\% patients had $\geq$ grade 3 adverse events.
\end{abstract}

Conclusion PARPi/anti-PD-1 is an effective and tolerable method for patients with advanced solid tumors, and BRCA1/2 is a potential biomarker.

Keywords PARP inhibitors $\cdot$ Anti-PD- $1 \cdot$ Tumors $\cdot$ Efficacy $\cdot$ Safety

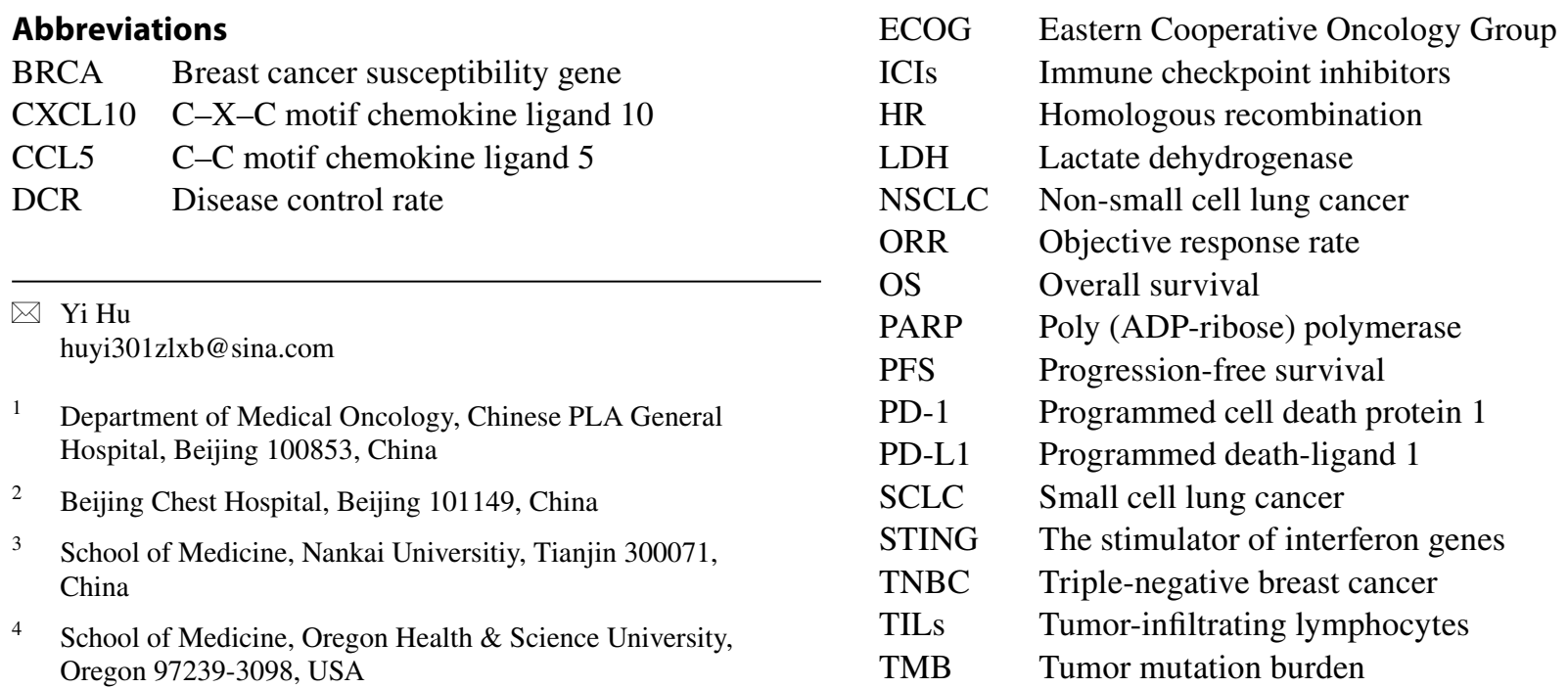




\section{Introduction}

In recent years, immune checkpoint inhibitors (ICIs) targeting PD-1/PD-L1 have achieved substantial advancements and been commonly used to treat different solid tumors. However, only a small portion of populations derive benefit, such as patients with high expression of programmed deathligand 1 (PD-L1), high tumor mutation burden (TMB) and microsatellite instability [1]. Programmed cell death protein 1(PD-1)/PD-L1 inhibitors can revive the exhausted T cells and enhance anticancer immune response. However, according to "the Cancer-Immunity Cycle" raised by Ira Mellman [2], elimination of tumor cells by $\mathrm{T}$ cells is only one of these steps. To reinvigorate the response and expand the potential benefit populations of anti-PD-1/PD-L1 blockades, combined therapies should be considered. ICIs in combination with agents that target other steps may be more effective, and PARP inhibitors belong to this kind of drugs with the ability to modulate tumor microenvironment.

PARP inhibitors are agents targeting homologous recombination (HR) pathway. PARP inhibitors, such as olaparib and niraparib, function to suppress cancer development by catalytic inhibition [3], PARP trapping [4] and modulation of anticancer immune response. The rationale for PARPi in combination with PD-1/PD-L1 inhibitors mainly involves four aspects: tumor neoantigen production, increasing tumor-infiltrating lymphocytes (TILs), enhanced antigen presentation and regulation of PD-L1 and other molecules in tumor microenvironment [5]. Different preclinical studies reported that PARP inhibitors significantly increased tumor infiltrating $\mathrm{CD} 4^{+} / \mathrm{CD}^{+}{ }^{+} \mathrm{T}$ cells which were activated by the stimulator of interferon genes (STING) pathway and recruited to tumors by tumor-specific neoantigens when combined with ICIs [6, 7]. And several preclinical studies showed that PARP inhibitors combined with antiPD-1/PD-L1 exhibited better efficacy compared with single agents, usually with up-regulation of PD-L1 via STING activation or GSK-3 $\beta$ inactivation $[6,8,9]$, and the enhanced therapeutic efficacy was independent of BRCA status [10]. It was also reported that PARP inhibition improved the number and killing activity of natural killer cells, accompanied by increased production of TNF- $\alpha$ and IFN- $\gamma[7,11]$.

Up to now, there have been many clinical studies about PARPi/ICIs underway, but available results are limited. The researchers of TOPACIO trial analyzed the efficacy and safety of niraparib/pembrolizumab combination therapy for patients with recurrent ovarian cancer and triplenegative breast cancer (TNBC), the ORRs were $18 \%$ and $21 \%$, and the DCRs were $65 \%$ and $49 \%$ [12, 13]. Olaparib/ durvalumab combination therapy displayed a clinical benefit rate of $21.1 \%(4 / 19)$ in patients with platinum-resistant SCLC [14], and it also showed notable efficacy for metastatic castration-resistant prostate cancer with 53\%(9/17) demonstrated a PSA declination more than 50\% [15]. These data demonstrate promising potentials for clinical application of PARPi/ICIs, in this study, we evaluated the efficacy and safety of olaparib/niraparib combined with pembrolizumab/ nivolumab in different advanced tumors and explored the potential biomarkers and possible resistant mechanisms.

\section{Method}

\section{Study design}

This retrospective study collected patients with advanced solid tumors who received one or more cycles of PARPi/ anti-PD1-based therapy between May 2017 and July 2019. Eligible patients were screened by the electronic medical record management system from the oncology department of People's Liberation Army General Hospital, according to the following criteria: (1) histologically confirmed metastatic solid tumors with measurable disease; (2) the response should be evaluable and survival status could be obtained by follow-up ; (3) PD-L1 expression, BRCA1/2 mutation and TMB data were available and convincing, or tissue samples could be provided to detect the above markers; (4) the PARPi/anti-PD1-based treatment therapy was discussed and decided by a multidisciplinary team (MDT). The detailed individual regimens are provided in supplementary Table 1.

To ensure data consistency, we prospectively designed our study protocol, case report form (CRF) and the standard operating procedure (SOP) of data collection. Available pre-treatment and post-two cycles treatment blood samples $(n=18)$ and tissue specimens $(n=25)$ were collected for next generation sequencing (NGS) and PD-L1 detection by immunohistochemistry (clone 22C3) after written informed consents were obtained.

\section{Data collection and study outcomes}

Clinicopathological information and treatment data were independently sorted and extracted by two physicians and all image materials were independently assessed and analyzed by two radiologists according to Response Evaluation Criteria in Solid Tumors, version 1.1 (RECIST 1.1), and all adverse events were recorded according to the National Cancer Institute Common Terminology Criteria for Adverse Events, version 4.0. The cutoff date of data was March 18, 2020. The efficacy and outcome measures of this study included ORR (the percentage of patients with CR/PR as per RECIST 1.1), DCR (the proportion of patients with CR/ $\mathrm{PR} / \mathrm{SD}$ as per RECIST 1.1), PFS (time from initial treatment to disease progression/death) and OS (time from initial treatment to death). All patients were evaluable for response, 
and patients failing to reach PFS and OS endpoints were censored on cutoff date.

\section{Statistical analysis}

Clinical characteristics, demographics and safety data were summarized via descriptive statistical analysis. Point estimates and two-sided 95\% CIs were provided for the analysis of ORR and DCR, and Fisher's exact test was applied to compare the difference of two groups. For survival analysis, the median value and two-sided 95\% CIs were obtained by Kaplan-Meier methods with a $\mathrm{P}$ value determined by the log-rank test. Single factor analysis and binary logistic regression were used to sort the biomarker of response. Univariate analysis and multivariate COX regression model were used to investigate the influence factor of PFS and OS. Two-sided $\mathrm{P}$ values were evaluated, and $P<0.05$ was considered statistically significant. All statistical analyses were performed using SPSS 20.0 software (IBM, SPSS, Chicago, IL, USA). TMB was calculated by maftools software (version 1.4.28). Pathway enrichment analysis was performed with g:Profiler, visualized with EnrichmentMap (Cytoscape) and interpreted using clusterMaker2 (Cytoscape).

\section{Results}

\section{Patient characteristics}

Between May 2017 and July 2019, 42 patients were screened, and 40 patients who met the eligibility criteria were included in this study. The median follow-up time was 289 (42-681) days, 21 patients permanently discontinued treatment because of disease progression, and 14 patients discontinued treatment due to toxicities. Of all the study populations, 13 patients were diagnosed with NSCLC, eight with SCLC, six with gynecologic tumors, four with pancreatic cancer, three with cholangiocarcinoma, two with prostate cancer, two with sarcoma, one with breast cancer and one with pleural mesothelioma. The median age was 59 years old (range, 38-81), and $26(65.0 \%)$ patients were men. All patients had at least one metastasis, and 13 (32.5\%) patients had an Eastern Cooperative Oncology Group (ECOG) performance score of 2 or 3 . Among the study populations, the median treatment line was three (range $1-6)$, with 4 (10\%) of 40 patients receiving this combination therapy as first-line treatment owing to BRCA1/2 mutation and personal condition. Baseline lymphocyte number, lactate dehydrogenase (LDH) level, PD-L1 expression, BRCA status and TMB data were collected. Twenty-five patients had available tissue specimens to confirm the PD-L1 expression and perform BRCA1/2-included NGS, and 17 patients had blood samples to evaluate the change of TMB before and after treatment. Characteristics data are summarized in Table 1.

\section{Efficacy}

As of March 18, 2020, all patients were evaluable, and 35 (87.5\%) PFS events and 27 (67.5\%) deaths had occurred. The integrate ORR was $27.5 \%$, with a median duration of response of $\geq 6.9 \mathrm{~m}$ (supplementary Figure 3). For four patients with BRCA1/2 mutation who received olaparib/ pembrolizumab-based combined therapy as first-line treatment (the diagnosis was SCLC, rhabdomyosarcoma, cholangiocarcinoma and pancreatic cancer, respectively), disease was well controlled with three patients achieving PR and one patient achieving SD. For 36 patients in $\geq$ secondline treatment, the ORR was $22.2 \%$ (95\% CI, 8.0-36.5\%), the DCR was $83.3 \%$ (95\% CI, 70.5-96.1\%). The ORR and DCR were $23.1 \%$ (95\% CI, 5.0-53.8\%) and 84.6\% (95\% CI, $54.6-96.1 \%$ ) for NSCLC, and $28.6 \%$ (95\% CI, 3.7-71.0\%) and $71.4 \%$ (95\% CI, 29.0-96.3\%) for SCLC, separately (Table 2). The mPFS of all patients, 36 patients in $\geq$ second-line treatment, 13 patients with NSCLC and seven with SCLC were $4.6 \mathrm{~m}$ (95\%CI, 2.5-6.0), $4.2 \mathrm{~m}$ (95\% CI, 2.4-6. 3), $4.5 \mathrm{~m}$ (95\% CI, 2.0-6.9) and 3.7m (95\%CI, 0.4-7.0), separately (Fig. 1a). The mOS were $9.4 \mathrm{~m} \mathrm{(95 \%} \mathrm{CI,} \mathrm{4.7-14.0),}$ $11.4 \mathrm{~m}(95 \% \mathrm{CI}, 7.6-15.3), 12.7 \mathrm{~m}(95 \% \mathrm{CI}, 3.4-22.0)$ and $5.4 \mathrm{~m}$ (95\%CI, 2.8-8.0), respectively (Fig. 1b).

We analyzed the potential biomarkers for efficacy and prognosis, factors with $P<0.1$ for single factor analysis were included in binary logistic regression, and potential clinical biomarkers (BRCA, PD-L1 and TMB) were also included regardless of $P$ values, and results of logistic regression showed BRCA1/2 mutation was positively correlated with ORR $(P=0.008)$ and $\mathrm{LDH} \geq 250 \mathrm{U} / \mathrm{L}$ was negatively correlated with the DCR $(P=0.018)$. Results of univariate analysis and multivariate COX regression model indicated that lymphocyte number, ECOG and LDH significantly influenced both PFS and OS $(P<0.05$, supplementary Table 2$)$. The ORR of BRCA+ patients was $60.0 \%$ versus $8.0 \%$ $(P=0.001)$ when compared with BRCA-, while the mPFS and mOS were $6.1 \mathrm{~m}$ versus $4.0 \mathrm{~m}(P=0.022$, Fig. $2 \mathrm{~g})$ and $9.4 \mathrm{~m}$ versus $11.8 \mathrm{~m}(P=0.867$, Fig. $2 \mathrm{~h})$. Although PD-L1 was not shown as a predictive factor for efficacy and outcomes in our univariate and multivariate analyses, we also did PD-L1-based subgroup analysis of PFS and OS, given that PD-L1 was reported as a biomarker of PD-1 inhibitors. Results showed that the survival curves separated at about 2.5 and 5 months, respectively, then remained non-overlapping throughout the follow-up period. Compared with PD-L $1<1 \%$ group, the mPFS $(6.1 \mathrm{~m}$ vs. $4.0 \mathrm{~m})$ and $\mathrm{mOS}$ ( $12.7 \mathrm{~m}$ vs. $8.9 \mathrm{~m}$ ) of $\mathrm{PD}-\mathrm{L} 1 \geq 1 \%$ group improved, still, there was no statistical difference $\left(P_{1}=0.096, P_{2}=0.181\right.$, supplementary Fig. 1). Other factors-based subgroup analysis data 
Table 1 Clinical data for all patients

\begin{tabular}{|c|c|c|c|c|}
\hline Characteristic & $\begin{array}{l}\text { All patients } \\
(n=40)\end{array}$ & $\begin{array}{l}\geq 2 \text { nd line } \\
(n=36)\end{array}$ & $\begin{array}{l}\text { NSCLC } \\
(n=13)\end{array}$ & $\begin{array}{l}\text { SCLC } \\
(n=7)\end{array}$ \\
\hline Median age (range), & $59(31-83)$ & $58(31-83)$ & $57(42-76)$ & $64(54-73)$ \\
\hline \multicolumn{5}{|l|}{ Gender, $n(\%)$} \\
\hline Male & $26(65.0)$ & $24(66.7)$ & $12(92.3)$ & $7(100.0)$ \\
\hline Female & $14(35.0)$ & $12(33.3)$ & $1(7.7)$ & $0(0.0)$ \\
\hline \multicolumn{5}{|l|}{ ECOG, $n(\%)$} \\
\hline $0-1$ & $27(67.5)$ & $23(63.9)$ & $9(69.2)$ & $3(42.9)$ \\
\hline$\geq 2$ & $13(32.5)$ & $13(36.1)$ & $4(30.8)$ & $4(57.1)$ \\
\hline \multicolumn{5}{|l|}{ Smoking history, $n(\%)$} \\
\hline Current or former & $17(42.5)$ & $15(41.7)$ & $6(46.2)$ & $6(85.8)$ \\
\hline Never & $23(57.5)$ & $21(58.3)$ & $7(53.8)$ & $1(14.2)$ \\
\hline \multicolumn{5}{|l|}{ Metastasis number, $n(\%)$} \\
\hline Number $<3$ & $28(70.0)$ & $25(69.4)$ & 11(84.6) & $3(42.9)$ \\
\hline Number $\geq 3$ & $12(30.0)$ & 11(30.6) & $2(15.4)$ & $4(57.1)$ \\
\hline \multicolumn{5}{|l|}{ BRCA mutation status, $n(\%)$} \\
\hline Mutation & $15(37.5)$ & $12(33.33)$ & $5(38.5)$ & $1(14.2)$ \\
\hline Wild-type & $25(62.5)$ & $24(66.67)$ & $8(61.5)$ & $6(85.8)$ \\
\hline \multicolumn{5}{|l|}{ PD-L1 expression, $n(\%)$} \\
\hline$<1 \%$ & $25(62.5)$ & $22(61.1)$ & $7(53.8)$ & $4(57.1)$ \\
\hline$\geq 1 \%$ & $15(37.5)$ & $14(38.9)$ & $6(46.2)$ & $3(42.9)$ \\
\hline TMB, median (range), n (\%) & $6.5(0-30)$ & $6.1(0-30)$ & $10.8(1.1-16.1)$ & $8.9(1.1-30)$ \\
\hline$<10 \mathrm{~m} / \mathrm{Mb}$ & $26(65.0)$ & $24(66.7)$ & $5(38.5)$ & $4(57.1)$ \\
\hline$\geq 10 \mathrm{~m} / \mathrm{Mb}$ & $14(35.0)$ & $12(33.3)$ & $8(61.5)$ & $3(42.9)$ \\
\hline \multicolumn{5}{|l|}{ Lymphocyte number, $n(\%)$} \\
\hline$<0.8 \times 10^{9} / \mathrm{L}$ & $14(30.5)$ & $12(33.3)$ & $4(30.8)$ & $2(28.6)$ \\
\hline$\geq 0.8 \times 10^{9} / \mathrm{L}$ & $26(65.0)$ & $24(66.7)$ & $9(69.2)$ & $5(71.4)$ \\
\hline \multicolumn{5}{|l|}{$\mathrm{LDH}, n(\%)$} \\
\hline$<250 \mathrm{U} / \mathrm{L}$ & $28(70.0)$ & $24(66.7)$ & $10(76.9)$ & $3(42.9)$ \\
\hline$\geq 250 \mathrm{U} / \mathrm{L}$ & $12(30.0)$ & $12(33.3)$ & $3(23.1)$ & $4(57.1)$ \\
\hline \multicolumn{5}{|l|}{ Treatment lines, $n(\%)$} \\
\hline $1-2$ & $16(40.0)$ & $12(33.3)$ & $2(18.2)$ & $5(71.4)$ \\
\hline$\geq 3$ & $24(60.0)$ & $24(66.7)$ & $11(81.8)$ & $2(28.6)$ \\
\hline \multicolumn{5}{|l|}{ Combined chemotherapy, $n(\%)$} \\
\hline Yes & $22(55.0)$ & $19(52.8)$ & $6(46.2)$ & $3(42.9)$ \\
\hline No & $18(45.0)$ & $17(47.2)$ & $7(53.8)$ & $4(57.1)$ \\
\hline Cycles of treatment, median (range), no. & $6(1-20)$ & $6(1-20)$ & $6(2-20)$ & $4(1-18)$ \\
\hline Follow-up time, median (range), days & $289(42-681)$ & $344(42-681)$ & $376(74-681)$ & $162(42-550)$ \\
\hline
\end{tabular}

NSCLC non-small cell lung cancer; SCLC small cell lung cancer; ECOG Eastern Cooperative Oncology Group; $P D-L 1$ programmed death-ligand 1; BRCA breast cancer susceptibility gene; $T M B$ tumor mutation burden; $L D H$ lactate dehydrogenase of ORR and DCR are summarized in supplementary Table 3, and lymphocyte number, ECOG, LDH and BRCA1/2-based subgroup analyses of PFS and OS are shown in Fig. 2. Since chemotherapy could also modulate immune response, we compared the outcomes of patients between PARPi/ICI/ chemotherapy group and PARPi/ICI group, and the results showed a mPFS of $4.5 \mathrm{~m}(95 \% \mathrm{CI}, 2.6-6.4 \mathrm{~m})$ versus $4.2 \mathrm{~m}(95 \% \mathrm{CI}, 1.0-7.5 \mathrm{~m})$, and a mOS of $8.9 \mathrm{~m}(95 \% \mathrm{CI}$, 5.2-12.6 m) versus $9.4 \mathrm{~m}(95 \% \mathrm{CI}, 0.0-19.4 \mathrm{~m})\left(P_{1}=0.695\right.$, $P_{2}=0.919$, supplementary Fig. 2).
Analysis of patients in $\geq$ second-line treatment was similar (data not shown), and multivariable analysis of NSCLC and SCLC was not conducted because of small case number. Univariate analysis of NSCLC also showed that patients with BRCA1/2 mutation had a higher ORR [60\% (95\%CI, $31.9-88.1 \%)$ vs. $0 \%$ (95\%CI, $0-36.9 \%), P=0.035]$, and patients with baseline lymphocyte $\geq 0.8 \times 10^{9} / 1$ had a better mPFS [8.7 m (95\%CI, 0-20.9) vs. $2.0 \mathrm{~m}(0.1-3.8)$, $P=0.021)$. 
Table 2 Best overall tumor responses in the full-analysis and efficacy-evaluable populations

\begin{tabular}{|c|c|c|c|c|c|c|}
\hline Populations & Total no. & CR/PR no.(\%) & SD no. $(\%)$ & PD no. $(\%)$ & ORR No.(\%) $[95 \% \mathrm{CI}]^{\mathrm{a}}$ & DCR No.(\%) $[95 \% \mathrm{CI}]^{\mathrm{b}}$ \\
\hline NSCLC & 13 & $3(23.1)$ & $8(61.5)$ & $2(15.4)$ & $23.1(5.0-53.8)$ & $84.6(54.6-96.1)$ \\
\hline SCLC $^{\mathrm{c}}$ & 8 & $3(28.6)$ & $3(37.5)$ & $2(28.6)$ & $28.6(3.7-71.0)$ & 71.4(29.0-96.3) \\
\hline Gynecologic tumors $^{\mathrm{d}}$ & 6 & 2 & 4 & 0 & 33.3 & 100.0 \\
\hline Pancreatic cancer & 4 & 1 & 3 & 0 & 25.0 & 100.0 \\
\hline Cholangiocarcinoma & 3 & 1 & 1 & 1 & 33.3 & 66.7 \\
\hline Others ${ }^{\mathrm{e}}$ & 6 & 1 & 5 & 0 & 16.7 & 83.3 \\
\hline First line & 4 & 3 & 1 & 0 & 75.0 & 100.0 \\
\hline$\geq$ Second line & 36 & $8(22.2)$ & $22(61.1)$ & $6(16.7)$ & $22.2(8.0-36.5)$ & $83.3(70.5-96.1)$ \\
\hline All populations & 40 & $11(27.5)$ & $23(57.5)$ & $6(15.0)$ & $27.5(13.0-42.0)$ & $85.0(73.4-96.6)$ \\
\hline
\end{tabular}

${ }^{a}$ Include complete and partial responses

${ }^{\mathrm{b}}$ Include complete and partial responses and stable disease

${ }^{c}$ Patient for first-line treatment( $\left.\mathrm{n}=1, \mathrm{PR}\right)$ was not included when calculated ORR and DCR

${ }^{\mathrm{d}}$ Include two cases of ovarian cancer, two of endometrial carcinoma, one of cervical carcinoma and one of fallopian tube cancer

${ }^{\mathrm{e}}$ Include two cases of prostate cancer, two of sarcoma, one of breast cancer and one of pleural mesothelioma

\section{$\Delta T M B$, molecular characteristics and possible resistance mechanisms}

We compared the $\triangle \mathrm{TMB}$ of 18 cases with response evaluation (supplementary Table S4), and we found patients with BRCA1/2 mutation had higher rate of $\Delta \mathrm{TMB}>0(66.7 \% \mathrm{vs}$. $11.1 \%, P=0.206)$. And subgroup analysis showed ORR of patients with $\triangle \mathrm{TMB}>0$ was higher than those with $\Delta \mathrm{TMB} \leq 0(66.7 \%$ vs. $20 \%, P=0.321)$, but there was no statistic difference maybe because of small case number.

We did a comparative pathway enrichment analysis between favorable and unfavorable-efficacy groups, by Gene Ontology (GO) molecular function database. Results showed that pathways enriched in two groups were different with 12 pathways overlapped, 29 pathways only in favorable-efficacy group and five pathways only in unfavorable-efficacy group. Further interpretation found that eight clusters were only enriched in favorable-efficacy group, including "purine binding ribonucleotide," "kinase enzyme binding," "protein phosphatase binding," "DNA binding," "signaling receptor binding," "protein complex binding," "identical protein binding" and "transducer activity signaling" (Fig. 3a), while five pathways were only enriched in unfavorable-efficacy group, including "VEGF binding," "VEGFR activity," "1-phophatidylinositol-4-phosphate-3-kinase activity," "neurotrophin receptor activity" and "transcription factor binding" (Fig. 3b).

We also summarized the molecular characteristics at disease progression and found the possible resistant mechanisms included sarcomatous degeneration and secondary gene mutation, of which one patient with pancreatic cancer was found to be with multiple BRCA2 truncation mutation,
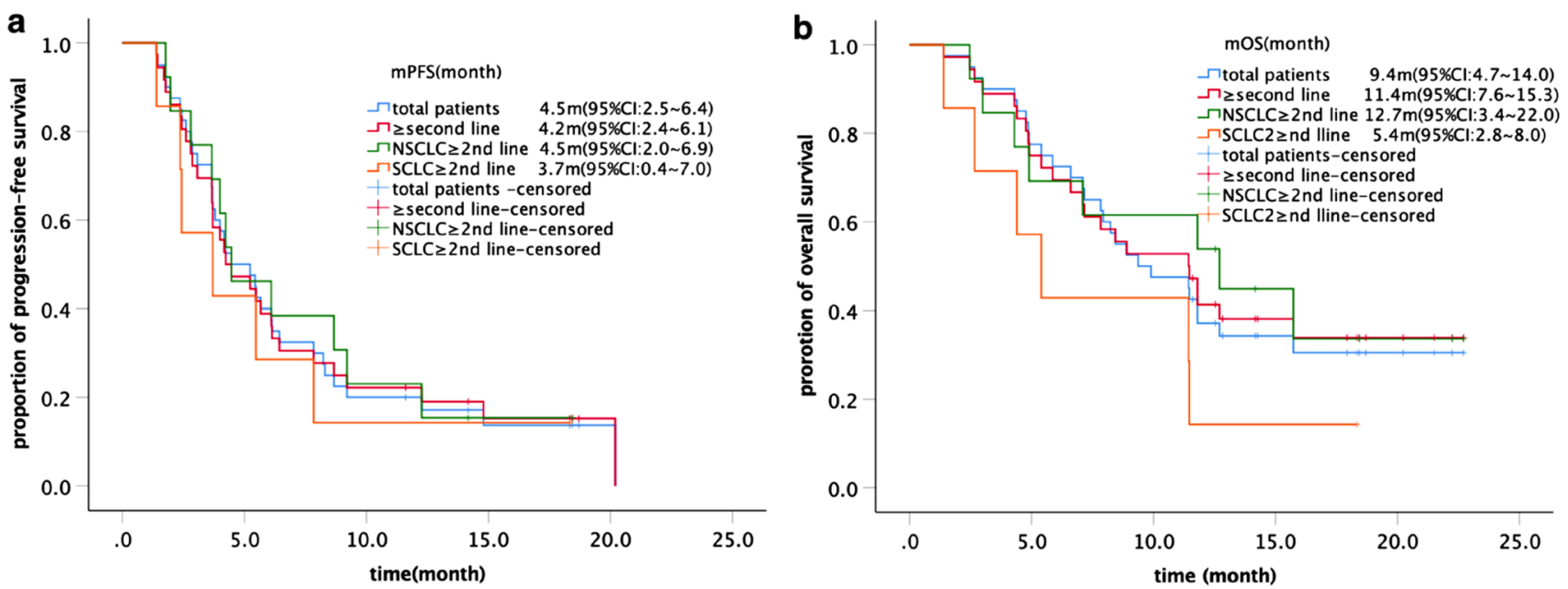

Fig. 1 The PFS (a) and OS (b) of total populations, $\geq 2$ nd line, NSCLC and SCLC 

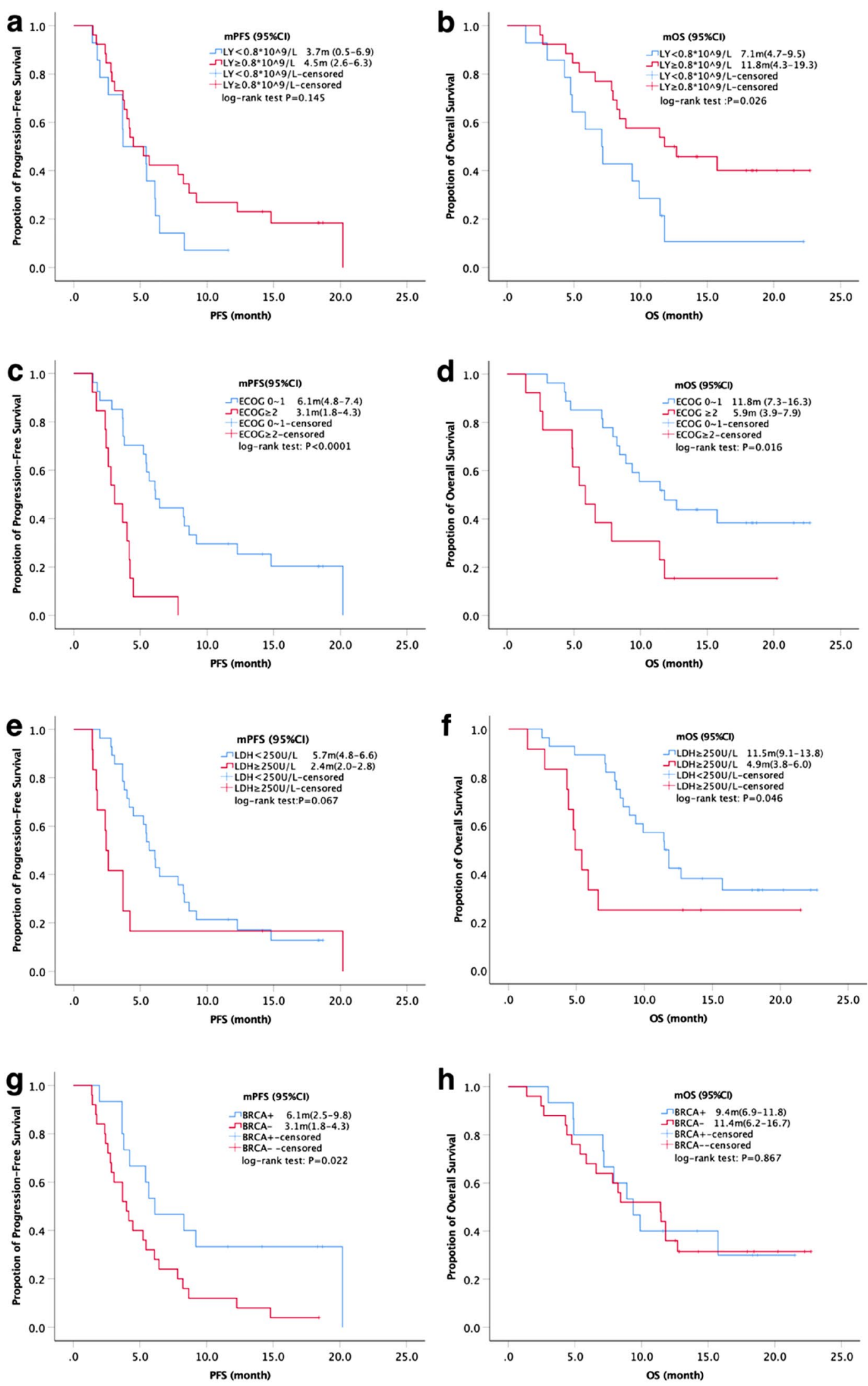

Fig. 2 Subgroup analysis of PFS and OS. Lymphocyte number-based subgroup analysis of PFS (a) and OS (b), ECOG-based subgroup analysis of PFS (c) and OS (d), LDH-based subgroup analysis of PFS (e) and OS (f), BRCA-based subgroup analysis of PFS (g) and OS (h) 


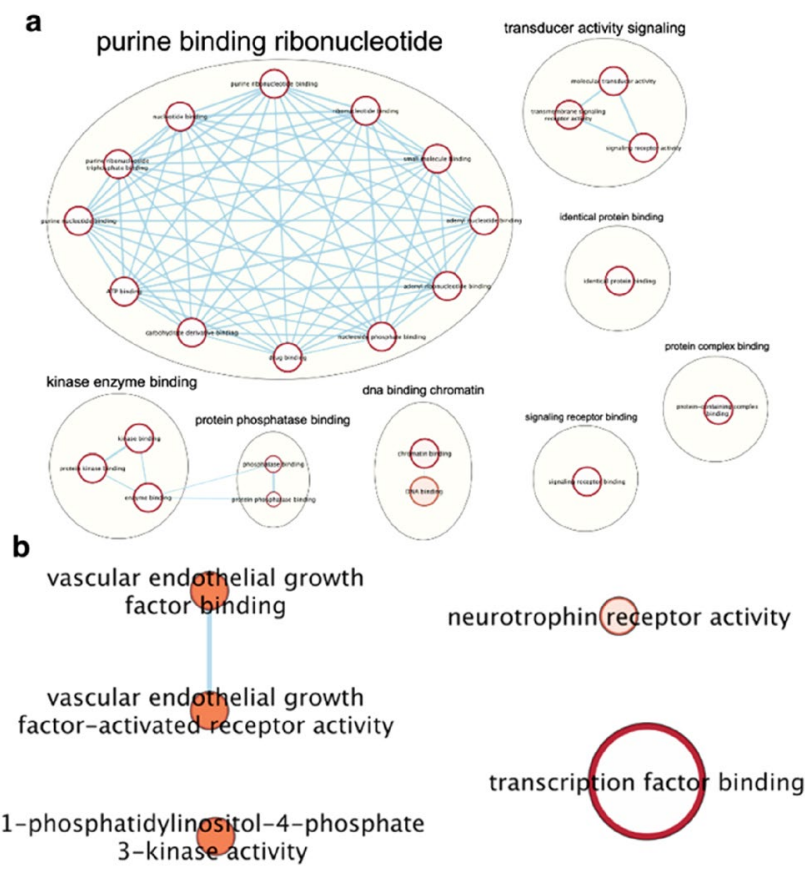

Fig. 3 The molecular function pathways enriched in favorable-efficacy group (a) and unfavorable-efficacy group (b). a Favorable-efficacy group includes patients with $\mathrm{PR} / \mathrm{CR}$ and $\mathrm{SD} \geq 6 \mathrm{~m}$; b Unfavorable-efficacy group includes patients with $\mathrm{PD}$ and $\mathrm{SD}<6 \mathrm{~m}$

two patients with A2M mutation (SCLC and sarcoma), one with JAK1 mutation (pancreatic cancer), T790M mutation (cervical cancer), KEAP1 (prostate cancer) and mTOR mutation (NSCLC), respectively.

\section{Safety}

The total incidence of adverse events was $95.0 \%$ (95\%CI, $83.1-99.4 \%)$, and 15 (37.5\%, 95\% CI, 22.7-54.2\%) of 40 patients had grade 3-5 treatment-related adverse events (TRAE), of whom one patient died of immune-related pneumonitis, three patients permanently discontinued treatment, and 10 patients temporarily suspended and re-challenged treatment after adverse effects were well-controlled. The overall rate of immune-related adverse events (irAEs) was $17.5 \%$, and incidence of $\geq$ grade 3 irAEs was $7.5 \%$. The most common TRAE were anemia (in 19 [47.5\%] of 40 patients), fatigue (9 $[22.5 \%])$, neutropenia (9 [22.5\%]), thrombopenia $(8[20 \%])$, loss of appetite $(8[20 \%])$, nausea and vomiting (8 [20\%]). And compared with chemo- group, the incidence of myelosuppression in chemo+ group was higher (77.3\% vs. $38.9 \%, P=0.023)$ (Table 3$)$.

\section{Discussion}

Given the ability of PARP inhibitors to modify the tumor microenvironment, especially the recruitment and priming of $\mathrm{CD}^{+}$and $\mathrm{CD} 8^{+} \mathrm{T}$ cells by producing neoantigen and releasing cytokines and chemokines, such as INF- $\gamma$, CCL5 and CXCL10 [10, 16], PD-1 inhibitors in combination with PARPi have potential to broaden durable responses and extend benefit populations of both PD-1 inhibitors and PARP inhibitors. Our study found that PARPi/anti-PD-1 combination therapy exhibited remarkable clinical efficacy in advanced solid tumors, especially BRCA1/2 mutant patients. For four patients in first-line treatment, three achieved PR and one achieved SD, and for 36 patients in $\geq$ second-line treatment, the ORR, DCR, mPFS and mOS were $22.2 \%$, $83.3 \%, 4.2 \mathrm{~m}$ and $11.4 \mathrm{~m}$. We also found that $28.6 \%(2 / 7)$ of patients with SCLC ( $\geq$ second line) and $33.3 \%(2 / 6)$ of patients with gynecologic tumors achieved $\mathrm{PR}$, which was higher than single anti-PD-1 regimen in the similar settings (13.7\% for SCLC [17] and $<15 \%$ for gynecologic tumors [18]). The ORRs of PARPi/anti-PD-1 in both SCLC and gynecologic tumors were a little higher than other studies $[12,14]$, maybe owing to earlier treatment line, combined chemotherapy and different population characteristics. Since PD-1 plus chemotherapy has been well studied in NSCLC, we have tried to compare the response rates of this combination with PARPi in addition to PD-1/chemotherapy, but different treatment lines lead to a limitation. Binary logistic regression analysis indicated that BRCA status was a biomarker for response, and subgroup analysis showed better ORR in BRCA+ group for both total efficacy-evaluable populations and NSCLC, which was in accordance with other study [13].

Survival analysis in our study showed longer mPFS but shorter mOS for BRCA+ when compared with BRCA-, however, statistic difference was seen only in mPFS (6.1 m vs. $3.1 \mathrm{~m}, P=0.022)$ but not $\mathrm{mOS}(9.4 \mathrm{~m}$ vs. 11.4 $\mathrm{m}, P=0.867)$, which might due to small case number and different cancer types between BRCA+ and BRCA - group. The subgroup analysis of PARPi/ICIs combination in other studies showed longer mPFS for BRCA+ in TNBC cohort instead of ovarian cancer $[12,13]$, and no data of mOS were reported, therefore, it will be hard to sort out if the difference is due to the biomarker or cancer types, and prospective studies with larger number of patients and specific cancer type are needed. Despite that results of binary logistic regression showed TMB had significant effect on ORR $(P=0.045)$, subgroup analysis by TMB showed no statistic difference ( $19.2 \%$ vs. $42.8 \%$, $P=0.148$ ), and there was no statistic difference of ORR between patients with $\Delta \mathrm{TMB}>0$ and $\Delta \mathrm{TMB} \leq 0$. We thought that small case number was a reason, and the time 
Table 3 Treatment-related adverse events of patients with or without chemotherapy

\begin{tabular}{|c|c|c|c|c|c|c|}
\hline \multirow[t]{2}{*}{ Adverse events } & \multicolumn{3}{|c|}{ Any grade no $(\%)$} & \multicolumn{3}{|c|}{ Grade $\geq 3$ No $(\%)$} \\
\hline & Total & Chemo+ & Chemo- & Total & Chemo+ & Chemo- \\
\hline Any treatment-related & $38(95.0)$ & $21(95.5)$ & $17(94.4)$ & a $14(35.0)$ & a $9(40.9)$ & $5(27.8)$ \\
\hline Immune-related adverse events & $7(17.5)$ & $3(13.6)$ & $4(22.2)$ & $3(7.5)$ & $1(4.5)$ & $2(11.1)$ \\
\hline${ }^{\mathrm{b}}$ Myelosuppression & $24(60.0)$ & $17(77.3)$ & $7(38.9)$ & $12(30.0)$ & $9(40.9)$ & $3(16.7)$ \\
\hline Anemia & $19(47.5)$ & $13(59.1)$ & $6(33.3)$ & $10(25.0)$ & $8(36.4)$ & $2(11.1)$ \\
\hline Leukopenia and/or neutropenia & $9(22.5)$ & $8(36.4)$ & $1(5.6)$ & $2(5.0)$ & $1(4.5)$ & $1(5.6)$ \\
\hline Thrombocytopenia & $8(20.0)$ & $7(31.8)$ & $1(5.6)$ & $3(7.5)$ & $2(9.1)$ & $1(5.6)$ \\
\hline Decreased appetite & $8(20.0)$ & $5(22.7)$ & $3(16.7)$ & $0(0.0)$ & $0(0.0)$ & $0(0.0)$ \\
\hline Nausea and vomiting & $8(20.0)$ & $6(27.3)$ & $2(11.1)$ & $0(0.0)$ & $0(0.0)$ & $0(0.0)$ \\
\hline Diarrhea & $4(10.0)$ & $1(4.5)$ & $3(16.7)$ & $1(2.5)$ & $0(0.0)$ & $0(0.0)$ \\
\hline Abdominal pain and discomfort & $3(7.5)$ & $2(9.1)$ & $1(5.6)$ & $0(0.0)$ & $0(0.0)$ & $0(0.0)$ \\
\hline Rash & $4(10.0)$ & $3(13.6)$ & $1(5.6)$ & $1(2.5)$ & $0(0.0)$ & $1(5.6)$ \\
\hline Myalgia and arthralgia & $3(7.5)$ & $2(9.1)$ & $1(5.6)$ & $0(0.0)$ & $0(0.0)$ & $0(0.0)$ \\
\hline Fever & $2(5.0)$ & $1(4.5)$ & $1(5.6)$ & $0(0.0)$ & $0(0.0)$ & $0(0.0)$ \\
\hline Fatigue & $9(22.5)$ & $3(13.6)$ & $6(33.3)$ & $0(0.0)$ & $0(0.0)$ & $0(0.0)$ \\
\hline Adenine increase & $2(5.0)$ & $1(4.5)$ & $1(5.6)$ & $0(0.0)$ & $0(0.0)$ & $0(0.0)$ \\
\hline Transaminase increase & $4(10.0)$ & $2(9.1)$ & $2(11.1)$ & $1(2.5)$ & $0(0.0)$ & $1(5.6)$ \\
\hline Hyperthyroidism & $1(2.5)$ & $0(0.0)$ & $1(5.6)$ & $0(0.0)$ & $0(0.0)$ & $0(0.0)$ \\
\hline Hypothyroidism & $2(5.0)$ & $2(9.1)$ & $0(0.0)$ & $0(0.0)$ & $0(0.0)$ & $0(0.0)$ \\
\hline Pneumonitis & $5(12.5)$ & $1(4.5)$ & $4(22.2)$ & $2(5.0)$ & $1(4.5)$ & $1(5.6)$ \\
\hline
\end{tabular}

${ }^{a}$ One death of immune-related pneumonitis was listed as grade 5 adverse events

${ }^{\mathrm{b}}$ Chemotherapy may increase the total incidence of myelosuppression at all grade level $(P=0.023)$ to collect blood samples should be adjusted. We also did PD-L1-based subgroup analysis of PFS and OS, although the survival curves separated and the MPFS and mOS of PD-L1 $\geq 1 \%$ group improved, still statistical difference was not found. Chemotherapy used to be considered to promote the response of ICIs to cancer [19], but in our study, chemotherapy-based subgroup analysis of PFS and OS showed no statistical difference, and this was in accordance with two recent studies, which indicated that chemotherapy weakly contributed to predicted neoantigen expression in ovarian cancer [20], and that chemotherapy exerted unfavorable influence on subsequent immunotherapy by inducing a decrease in tumor mutation burden [21]. Therefore, the effect of chemotherapy on ICIs should be further studied. In addition, maybe PARP inhibitors synergized with PD-1 blockade mainly by regulating the immune context, especially TILs, and in our study, baseline lymphocyte was found to markedly influence both PFS and OS, therefore, further dynamic detection of lymphocyte immunophenotyping is important.

We did molecular function pathway enrichment analysis with GO database, and VEGF/VEGFR pathways were found enriched in unfavorable-efficacy group, and this provided an insight for us to appropriately combine PARPi/antiPD-1 with anti-angiogenesis drugs according to patients' clinical condition. Meanwhile, favorable-efficacy group was more enriched in purine/DNA/kinase/transducer signaling receptor binding associated pathways, and it was in accordance with "BRCA mutation was a biomarker for response," therefore, it is worthy to explore if it is feasible to add platinum to this combined therapy in BRCA1/2 wild-type tumors to increase DNA damage pressure. Besides, some of the patients conducted NGS at the time of PD, and we identified distinct gene mutation compared with pre-treatment, such as secondary BRCA1/2 truncation mutation, A2M, JAK1, KEAP1, T790M and mTOR, of which BRCA2 truncation mutation was reported to be an important resistance mechanism of PARP inhibitors [22], and JAK1 truncating mutation was recognized as an acquired resistance mechanism of PD-1 inhibitors owing to a lack of response to interferon gamma [23]. A2M mutation was related to inflammatory cascades and might facilitate cancer development by decreasing the expression of CD29 and CD44 [24], in addition, T790M, mTOR mutation and KEAP1 loss were identified as resistance mechanisms [25]. However, our small study only verified that multiple secondary BRCA2 mutation was a resistance mechanism of PARPi/PD-1 blockade therapy [26], other putative mechanisms should be further verified.

In the perspective of safety, the total adverse events were similar with other studies [12,13], and the irAEs did not increase compared with ICIs [27], but the incidence of myelosuppression was a little higher, which was probably due to the additional utilization of chemotherapy. Based 
on our data and other clinical investigations, we suggest that PARPi/ICIs-related clinical trial is a consideration for advanced tumors without standard treatment, especially for those selected patient population, such as BRCA1/2 mutation. Still, there were some limitations in our study, for example, we did not distinguish germline or somatic mutation, because there were only two cases with germline BRCA1/2 mutation, so BRCA mutation forms and other DNA damage-related mutations, particularly HR deficiency, should also be considered. Meanwhile, combination treatment drugs in our study were not entirely the same, and part of PD-L1 expression and NGS data was collected from other platforms. In addition, comparison with PD-1 inhibitors alone should be further done. Therefore, prospective clinical studies are needed to better understand the anti-tumor effect of PARPi/anti-PD-1 combination, and further analysis of biomarker and immunophenotyping is important.

Supplementary Information The online version of this article (https:// doi.org/10.1007/s00262-021-02852-4) contains supplementary material, which is available to authorized users.

Acknowledgements We thank the patients and their families, and Professor Qian for linguistic polish, and we thank Genetron Health (Beijing) Co. Ltd. for molecular detection support

Authors' contributions All authors contributed to the study conception and design. ZZW, HTT and SJZ collected the data. ZZW analyzed the data, XW did pathway enrichment analysis. ZZW drafted the manuscript, and the other authors revised the manuscript. All authors have reviewed and approved the final manuscript.

Data and Code availability Not applicable.

\section{Compliance with ethical standards}

Conflict of interest The authors declare that there is no conflict of interest.

Ethics approval This retrospective study involving human participants was in accordance with the ethical standards of the national research committee and with the Helsinki Declaration. This study was approved by the ethics committee of Chinese PLA General Hospital (approval number: S2019-184-01)

Consent All the patients were informed of the possible treatmentrelated adverse events by doctors and signed informed consent before using PARP inhibitor and PD-1/PD-L1 inhibitor. Written informed consent was obtained before blood samples and tissue specimens were collected. This study does not contain any individual person's information of patients, and all patients have consented to participate in this study and publish this paper.

Open Access This article is licensed under a Creative Commons Attribution 4.0 International License, which permits use, sharing, adaptation, distribution and reproduction in any medium or format, as long as you give appropriate credit to the original author(s) and the source, provide a link to the Creative Commons licence, and indicate if changes were made. The images or other third party material in this article are included in the article's Creative Commons licence, unless indicated otherwise in a credit line to the material. If material is not included in the article's Creative Commons licence and your intended use is not permitted by statutory regulation or exceeds the permitted use, you will need to obtain permission directly from the copyright holder. To view a copy of this licence, visit http://creativecommons.org/licenses/by/4.0/.

\section{References}

1. Havel JJ, Chowell D, Chan TA (2019) The evolving landscape of biomarkers for checkpoint inhibitor immunotherapy. Nat Rev Cancer 19(3):133-150. https://doi.org/10.1038/s41568-019-0116-x

2. Chen DS, Mellman I (2013) Oncology meets immunology: the cancer-immunity cycle. Immunity 39(1):1-10. https://doi.org/10. 1016/j.immuni.2013.07.012

3. Lord CJ, Ashworth A (2017) PARP inhibitors: Synthetic lethality in the clinic. Science 355(6330):1152-1158. https://doi.org/10. 1126/science.aam7344

4. Murai J, Huang SY, Das BB, Renaud A, Zhang Y, Doroshow JH, Ji J, Takeda S, Pommier Y (2012) Trapping of PARP1 and PARP2 by clinical PARP inhibitors. Cancer Res 72(21):55885599. https://doi.org/10.1158/0008-5472.CAN-12-2753

5. Stewart RA, Pilie PG, Yap TA (2018) Development of PARP and immune-checkpoint inhibitor combinations. Cancer Res 78(24):6717-6725. https://doi.org/10.1158/0008-5472. CAN-18-2652

6. Wang Z, Sun K, Xiao Y, Feng B, Mikule K, Ma X, Feng N, Vellano CP, Federico L, Marszalek JR, Mills GB, Hanke J, Ramaswamy S, Wang J (2019) Niraparib activates interferon signaling and potentiates anti-PD-1 antibody efficacy in tumor models. Scientific Reports 9(1):1853. https://doi.org/10.1038/ s41598-019-38534-6

7. Huang J, Wang L, Cong Z, Amoozgar Z, Kiner E, Xing D, Orsulic S, Matulonis U, Goldberg MS (2015) The PARP1 inhibitor BMN 673 exhibits immunoregulatory effects in a Brca1(-/-) murine model of ovarian cancer. Biochem Biophys Res Commun 463(4):551-556. https://doi.org/10.1016/j.bbrc.2015.05.083

8. Jiao S, Xia W, Yamaguchi H, Wei Y, Chen MK, Hsu JM, Hsu JL, Yu WH, Du Y, Lee HH, Li CW, Chou CK, Lim SO, Chang SS, Litton J, Arun B, Hortobagyi GN, Hung MC (2017) PARP inhibitor upregulates PD-L1 expression and enhances cancer-associated immunosuppression. Clin Cancer Res Official J Am Assoc Cancer Res 23(14):3711-3720. https://doi.org/10.1158/1078-0432. CCR-16-3215

9. Robillard L, Nguyen M, Loehr A, Orsulic S, Kristeleit RS, Lin K, Raponi M, Harding TC, Simmons AD, Robillard L (2017) Preclinical evaluation of the PARP inhibitor rucaparib in combination with PD-1 and PD-L1 inhibition in a syngeneic BRCA1 mutant ovarian cancer model. In: Aacr Meeting.

10. Shen J, Zhao W, Ju Z, Wang L, Peng Y, Labrie M, Yap TA, Mills GB, Peng G (2019) PARPi triggers the STING-dependent immune response and enhances the therapeutic efficacy of immune checkpoint blockade independent of BRCAness. Cancer Res 79(2):311319. https://doi.org/10.1158/0008-5472.CAN-18-1003

11. Fenerty KE, Padget M, Wolfson B, Gameiro SR, Su Z, Lee JH, Rabizadeh S, Soon-Shiong P, Hodge JW (2018) Immunotherapy utilizing the combination of natural killer- and antibody dependent cellular cytotoxicity (ADCC)-mediating agents with poly (ADPribose) polymerase (PARP) inhibition. J Immunother Cancer 6(1):133. https://doi.org/10.1186/s40425-018-0445-4

12. Konstantinopoulos PA, Waggoner S, Vidal GA, Mita M, Moroney JW, Holloway R, Van Le L, Sachdev JC, Chapman-Davis E, Colon-Otero G, Penson RT, Matulonis UA, Kim YB, Moore KN, Swisher EM, Farkkila A, D’Andrea A, Stringer-Reasor E, Wang 
J, Buerstatte N, Arora S, Graham JR, Bobilev D, Dezube BJ, Munster P (2019) Single-arm phases 1 and 2 trial of Niraparib in combination with pembrolizumab in patients with recurrent platinum-resistant ovarian carcinoma. JAMA Oncol. https://doi. org/10.1001/jamaoncol.2019.1048

13. Vinayak S, Tolaney SM, Schwartzberg L, Mita M, McCann G, Tan AR, Wahner-Hendrickson AE, Forero A, Anders C, Wulf GM, Dillon P, Lynce F, Zarwan C, Erban JK, Zhou Y, Buerstatte N, Graham JR, Arora S, Dezube BJ, Telli ML (2019) Open-label clinical trial of Niraparib combined with Pembrolizumab for treatment of advanced or metastatic triple-negative breast cancer. JAMA Oncol. https://doi.org/10.1001/jamaoncol.2019.1029

14. Thomas A, Vilimas R, Trindade C, Erwin-Cohen R, Roper N, Xi L, Krishnasamy V, Levy E, Mammen A, Nichols S, Chen Y, Velcheti V, Yin F, Szabo E, Pommier Y, Steinberg SM, Trepel JB, Raffeld M, Young HA, Khan J, Hewitt S, Lee JM (2019) Durvalumab in Combination with Olaparib in Patients with Relapsed SCLC: Results from a Phase II Study. J Thoracic Oncol Official Publ Int Assoc Study Lung Cancer. https://doi.org/10.1016/j.jtho. 2019.04.026

15. Karzai F, Madan RA, Owens H, Couvillon A, Hankin A, Williams M, Bilusic M, Cordes LM, Trepel JB, Killian K, Meltzer PS, Gulley JL, Lee J-m, Dahut WL (2018) A phase 2 study of olaparib and durvalumab in metastatic castrate-resistant prostate cancer (mCRPC) in an unselected population. J Clinical Oncol 36 (6_suppl):163-163. https://doi.org/10.1200/JCO.2018.36.6_suppl. 163

16. Rosado MM, Bennici E, Novelli F, Pioli C (2013) Beyond DNA repair, the immunological role of PARP-1 and its siblings. Immunology 139(4):428-437. https://doi.org/10.1111/imm.12099

17. Reck M, Vicente D, Ciuleanu T, Gettinger S, Peters S, Horn L, Audigier-Valette C, Pardo N, Juan-Vidal O, Cheng Y (2018) Efficacy and safety of nivolumab (nivo) monotherapy versus chemotherapy (chemo) in recurrent small cell lung cancer (SCLC): Results from CheckMate 331. Ann Oncol Official J Europ Soc Med Oncol 29 (suppl_10):39-43. https://doi.org/10.1093/annonc/ mdy511.004

18. Garcia C, Ring KL (2018) The role of PD-1 checkpoint inhibition in gynecologic malignancies. Curr Treat Options Oncol 19(12):70. https://doi.org/10.1007/s11864-018-0593-2

19. Yu WD, Sun G, Li J, Xu J, Wang X (2019) Mechanisms and therapeutic potentials of cancer immunotherapy in combination with radiotherapy and/or chemotherapy. Cancer Lett 452:66-70. https://doi.org/10.1016/j.canlet.2019.02.048

20. O'Donnell T, Christie EL, Ahuja A, Buros J, Aksoy BA, Bowtell DDL, Snyder A, Hammerbacher J (2018) Chemotherapy weakly contributes to predicted neoantigen expression in ovarian cancer. BMC Cancer 18(1):87. https://doi.org/10.1186/ s12885-017-3825-0

21. He Y, Li X, Dang S, Liu G, Song L, Zhu B, Zhou C (2020) Effect of genomic and transcriptional alterations in first-line chemotherapy on subsequent immunotherapy in non-small cell lung cancer (NSCLC) patients. J Clin Oncol 38:9614-9614. https://doi.org/ 10.1200/JCO.2020.38.15_suppl.9614
22. Kondrashova O, Nguyen M, Shield-Artin K, Tinker AV, Teng NNH, Harrell MI, Kuiper MJ, Ho GY, Barker H, Jasin M, Prakash R, Kass EM, Sullivan MR, Brunette GJ, Bernstein KA, Coleman RL, Floquet A, Friedlander M, Kichenadasse G, O'Malley DM, Oza A, Sun J, Robillard L, Maloney L, Bowtell D, Giordano H, Wakefield MJ, Kaufmann SH, Simmons AD, Harding TC, Raponi M, McNeish IA, Swisher EM, Lin KK, Scott CL, Group AS (2017) Secondary somatic mutations restoring RAD51C and RAD51D associated with acquired resistance to the PARP inhibitor Rucaparib in high-grade ovarian carcinoma. Cancer Discovery 7(9):984-998. https://doi.org/10.1158/2159-8290.CD-17-0419

23. Zaretsky JM, Garcia-Diaz A, Shin DS, Escuin-Ordinas H, Hugo W, Hu-Lieskovan S, Torrejon DY, Abril-Rodriguez G, Sandoval S, Barthly L, Saco J, Homet Moreno B, Mezzadra R, Chmielowski B, Ruchalski K, Shintaku IP, Sanchez PJ, Puig-Saus C, Cherry G, Seja E, Kong X, Pang J, Berent-Maoz B, Comin-Anduix B, Graeber TG, Tumeh PC, Schumacher TN, Lo RS, Ribas A (2016) Mutations associated with acquired resistance to PD-1 blockade in melanoma. New Engl J Med 375(9):819-829. https://doi.org/ 10.1056/NEJMoa1604958

24. Kurz S, Thieme R, Amberg R, Groth M, Jahnke HG, Pieroh P, Horn LC, Kolb M, Huse K, Platzer M, Volke D, Dehghani F, Buzdin A, Engel K, Robitzki A, Hoffmann R, Gockel I, Birkenmeier $\mathrm{G}$ (2017) The anti-tumorigenic activity of A2M-A lesson from the naked mole-rat. PloS one 12(12):e0189514. https://doi.org/ 10.1371/journal.pone.0189514

25. Yu HA, Suzawa K, Jordan E, Zehir A, Ni A, Kim R, Kris MG, Hellmann MD, Li BT, Somwar R, Solit DB, Berger MF, Arcila M, Riely GJ, Ladanyi M (2018) Concurrent alterations in EGFR-mutant lung cancers associated with resistance to EGFR kinase inhibitors and characterization of MTOR as a mediator of resistance. Clin Cancer Res Official J Am Assoc Cancer Res 24(13):3108-3118. https://doi.org/10.1158/1078-0432. CCR-17-2961

26. Tao H, Liu S, Huang D, Han X, Wu X, Shao YW, Hu Y (2020) Acquired multiple secondary BRCA2 mutations upon PARPi resistance in a metastatic pancreatic cancer patient harboring a BRCA2 germline mutation. Am J Translational Res 12(2):612-617

27. Wang Y, Zhou S, Yang F, Qi X, Wang X, Guan X, Shen C, Duma N, Vera Aguilera J, Chintakuntlawar A, Price KA, Molina JR, Pagliaro LC, Halfdanarson TR, Grothey A, Markovic SN, Nowakowski GS, Ansell SM, Wang ML (2019) Treatment-related adverse events of PD-1 and PD-L1 inhibitors in clinical trials: a systematic review and meta-analysis. JAMA Oncol 5(7):10081019. https://doi.org/10.1001/jamaoncol.2019.0393

Publisher's Note Springer Nature remains neutral with regard to jurisdictional claims in published maps and institutional affiliations. 